\title{
Paraspidodera uncinata (Nematoda, Lauroiinae) como parasita de Cavia magna e Cavia aperea (Rodentia, Caviidae) no Sul do Brasil
}

\author{
Ana Gabriela da Silva Rocha ${ }^{1 *}$ \\ Moisés Gallas ${ }^{1}$ \\ Eliane Fraga da Silveira ${ }^{1}$ \\ Eduardo Périco ${ }^{2}$ \\ ${ }^{1}$ Laboratório de Zoologia de Invertebrados, Museu de Ciências Naturais \\ Universidade Luterana do Brasil, CEP 92425-900, Canoas - RS, Brasil \\ ${ }^{2}$ Laboratório de Ecologia, Museu de Ciências Naturais, Centro Universitário UNIVATES. 95900-000, Lajeado - RS, Brasil \\ * Autor para correspondência \\ anagabrieladasilvarocha@yahoo.com.br
}

Submetido em 09/09/2014

Aceito para publicação em 07/02/2015

\section{Resumo}

Das cinco espécies de preás registradas para o Brasil, quatro (Cavia fulgida, C. porcellus, C. aperea e C. magna) já foram registradas como hospedeiras de Paraspidodera uncinata. Os roedores C. magna e C. aperea são mamíferos pequenos, de hábitos terrestres, que ocorrem da região sul do Brasil ao leste do Uruguai. Preás coletadas e doadas foram necropsiados para análise de endoparasitos e os nematoides encontrados foram identificados como $P$. uncinata. Os microhábitats dessa espécie foram os intestinos delgado e grosso em $C$. magna e o intestino grosso em C. aperea. As prevalências de $P$. uncinata nos diferentes hospedeiros foram $60 \%$ $(C$. magna $; \mathrm{n}=5)$ e $14 \%(C$. aperea $; \mathrm{n}=7)$. O registro de $P$. uncinata em diferentes espécies de preás permite inferir que o modo de forrageio é semelhante nas diferentes localidades onde ocorrem no sul do Brasil, pois, possivelmente, a infecção dos hospedeiros ocorreu através da ingestão de vegetais com ovos de $P$. uncinata. Este trabalho contribui com o conhecimento sobre a helmintofauna de C. magna e C. aperea, no sul do Brasil.

Palavras-chave: Helmintofauna; Nematoides; Preá; Região Neotropical; Taxonomia

\section{Abstract}

Paraspidodera uncinata (Nematoda, Lauroiinae) as parasite of Cavia magna and Cavia aperea (Rodentia, Caviidae) in southern Brazil. Out of the 5 species of Guinea pig registered for Brazil, 4 (Cavia fulgida, C. porcellus, C. aperea, and C. magna) have already been reported as hosts of Paraspidodera uncinata. The rodent species $C$. magna and $C$. aperea are small-sized mammals, with terrestrial habits, which occur from southern Brazil to eastern Uruguay. Guinea pig specimens donated for research were necropsied for the analysis of endoparasites, and the nematodes found were identified as $P$. uncinata. The microhabitats of this species were the small and large intestines in C. magna and the large intestine in C. aperea. The prevalence of $P$. uncinata in the hosts was $60 \%$ in C. magna $(\mathrm{n}=5)$ and $14 \%$ in C. aperea $(\mathrm{n}=7)$. The record of $P$. uncinata in different Guinea pig species allows inferring that the foraging mode is similar in the different locations where they occur 
in southern Brazil, because, perhaps, the infection of hosts has occurred through the ingestion of vegetables contaminated with $P$. uncinata eggs. This study contributes to knowledge on the helminth fauna of $C$. magna and C. aperea in southern Brazil.

Key words: Guinea pig; Helminth fauna; Nematodes; Neotropical Region; Taxonomy

\section{Introdução}

No Brasil, ocorrem cinco espécies do gênero Cavia Pallas, 1766 (Cavia aperea Erxleben, 1777, Cavia fulgida Wagler, 1831, Cavia intermedia Cherem, Olímpio \& Ximenez, 1999, Cavia magna Ximenez, 1980 e Cavia porcellus Linnaeus, 1758) (OLIVEIRA; BONVICINO, 2011). Para o estado do Rio Grande do Sul, foram registradas C. aperea, C. magna (nativas) e C. porcellus (exótica) (OLIVEIRA; BONVICINO, 2011; CHEREM; FERIGOLO, 2012; FERNANDES et al., 2014). As espécies de Cavia têm hábito terrestre, habitam bordas de mata em áreas de Mata Atlântica e formações próximas a cursos d'água como mata de galeria, campo úmido, brejo, campo limpo no Cerrado, Campos do Sul e áreas da Caatinga (OLIVEIRA; BONVICINO, 2011).

Em relação à helmintofauna das espécies de Cavia no Brasil, já foram registrados dois trematódeos digenéticos (KAWAZOE et al., 1981; REY, 1993), dois cestoides (REGO, 1961; PINTO et al., 2002) e seis nematoides, dentre eles, Paraspidodera uncinata (Rudolphi, 1819) Travassos, 1914 (VICENTE et al., 1997).

Na América do Sul, P. uncinata possui ampla distribuição, sendo registrada como endoparasita para as famílias Caviidae, Echimyidae Gray, 1825, Cuniculidae Miller \& Gidley, 1918 e Octodontidae Waterhouse, 1839 no Peru (IBÁÑEZ, 1967; DITTMAR, 2002), na Bolívia (LENT; FREITAS, 1939 apud ROSSIN et al., 2004), na Argentina (PARONA, 1900; SUTTON, 1976; ROSSIN et al., 2004; ROBLES et al., 2012), no Brasil (TRAVASSOS, 1914; PEREIRA; VAZ, 1933; LENT; FREITAS, 1939 apud ROSSIN et al., 2004; PINTO et al., 1982; 2002; VICENTE et al., 1982; RIBEIRO et al., 1985; ALMEIDA, 2004; KRÜGER, 2006; SIMÕES et al., 2010) e no Uruguai (KHALIL; VOGELSANG, 1931a; 1931b; LENT; FREITAS, 1948).

No Brasil, P. uncinata foi registrada em $C$. aperea (TRAVASSOS, 1914; LENT; FREITAS, 1939 apud ROSSIN et al., 2004; PINTO et al., 1982; KRÜGER,
2006), C. porcellus (TRAVASSOS, 1914; PEREIRA; VAZ, 1933; LENT; FREITAS, 1939 apud ROSSIN et al., 2004; RIBEIRO et al., 1985; PINTO et al., 2002), Kerodon rupestris Wied-Neuwied, 1820 (LENT; FREITAS, 1939 apud ROSSIN et al., 2004; ALMEIDA, 2004), Cuniculus paca (Linnaeus, 1766) (TRAVASSOS, 1914; LENT; FREITAS, 1939 apud ROSSIN et al., 2004) e Thrichomys pachyurus (Wagner, 1845) (SIMÕES et al., 2010). Dentre esses registros, para o estado do Rio Grande do Sul, apenas Ribeiro et al. (1985) e Krüger (2006) realizam os estudos com $C$. aperea. Considerando que neste estado ocorrem apenas duas espécies nativas de Cavia este trabalho tem como objetivo registrar pela primeira vez o endoparasito $P$. uncinata em C. magna cujo conhecimento sobre ectoparasitos e endoparasitos é inexistente. Além disso, são relacionados dados ecológicos dos parasitos entre as duas espécies nativas de roedores.

\section{Material e Métodos}

Entre 2006 e 2009, espécimes de C. magna $(\mathrm{n}=5) \mathrm{e}$ C. aperea $(\mathrm{n}=7)$ encontrados mortos por atropelamento foram coletados e doados para o Laboratório de Zoologia de Invertebrados do Museu de Ciências Naturais, ULBRA (MCNU). Os espécimes de C. magna foram procedentes da Ilha dos Marinheiros, Rio Grande; C. aperea foram coletados nos municípios de Maratá $(\mathrm{n}=1)$, Pelotas e Bagé (BR 116) $(\mathrm{n}=6), \mathrm{RS}$, Brasil (Figura 1). A coleta dos preás foi realizada de acordo com a instrução normativa no 154 do IBAMA (MMA, 2007). Os nematoides $(n=17)$ encontrados no intestino dos hospedeiros foram fixados em A.F.A. $\left(65^{\circ} \mathrm{C}\right)$ por $48 \mathrm{~h}$ e, posteriormente conservados em etanol $70^{\circ} \mathrm{GL}$ (AMATO; AMATO, 2010). Alguns espécimes foram montados temporariamente em lâminas com lactofenol de Amann (HUMASON, 1979) para a identificação. A sistemática e classificação para os helmintos seguiram Chabaud (2009). Os parâmetros ecológicos das infecções foram calculados segundo Bush et al. (1997). 
FIGURA 1: Mapa com detalhe do estado do Rio Grande do Sul, indicando os locais de coleta de Cavia aperea Erxleben, 1777 (círculos) e Cavia magna Ximenez, 1980 (triângulo).

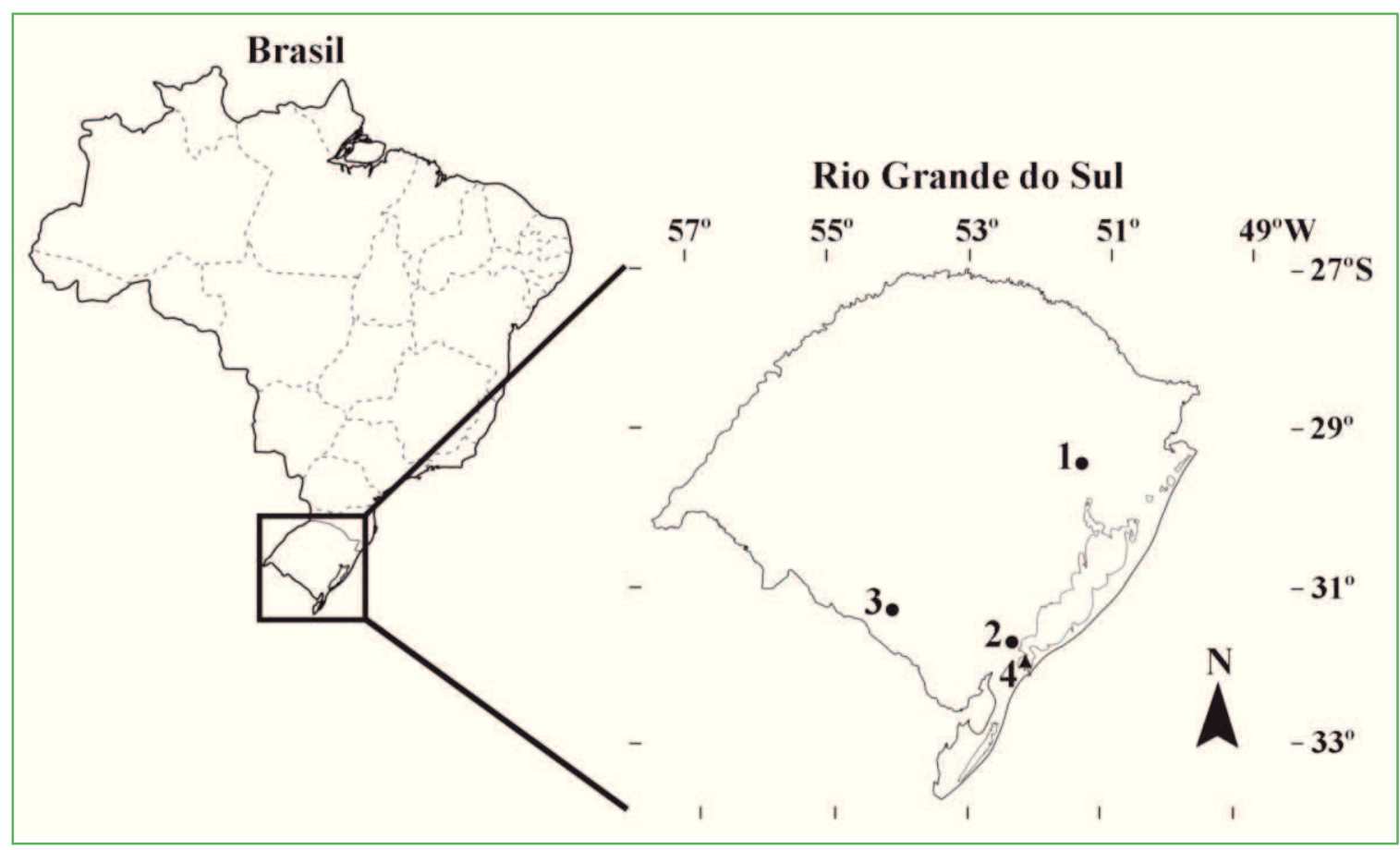

Locais de coleta: 1 - Maratá; 2 - Pelotas; 3 - Bagé; 4 - Ilha dos Marinheiros, Rio Grande.

As medidas morfométricas estão em micrômetros $(\mu \mathrm{m})$ e, caso contrário, a unidade foi indicada. Média, desvio padrão e número de espécimes medidos estão representados nesta ordem entre parênteses. As fotomicrografias foram realizadas com a utilização de uma câmera Sony DSC - S650. Um espécime representativo de cada espécie de hospedeiro foi depositado na Coleção de Mamíferos do Museu de Ciências Naturais da ULBRA (MCNU) Canoas, RS, Brasil. Espécimes representativos de nematoides foram depositados na Coleção Helmintológica do Museu de Ciências Naturais da ULBRA (CHMU) Canoas, RS, Brasil.

\section{Resultados}

Foram coletados 17 espécimes de $P$. uncinata (Figuras 2-5) (Aspidoderidae, Lauroiinae) em C. aperea $(\mathrm{n}=7)$ e $C$. magna $(\mathrm{n}=5)$. A descrição dos nematoides foi baseada em espécimes coletados de $C$. aperea $(\mathrm{n}=$ 2) e C. magna $(\mathrm{n}=6)$. Os caracteres morfológicos que permitiram a determinação foram: corpo alongado, com extremidade anterior com três lábios, um dorsal e dois ventrolaterais. Cutícula com estrias transversais. Esôfago com bulbo na extremidade posterior, correspondendo a $7,4 \%$ do comprimento total do corpo (CTC).

Machos ( $\mathrm{n}=2)$. Corpo com 11,2 e 11,4 mm (11,3 $\pm 0,14 ; 2)$ de comprimento, 239,72 e 258,16 (248,94 \pm $13,03 ; 2)$ de largura. Lábios com 55,2 e 64,4 (59,93 \pm $6,51 ; 2)$ de comprimento, 92,2 e 101,42 $(96,81 \pm 6,51$; 2) de largura. Poro excretor 552,24 e 623,04 (587,64 $\pm 50,06 ; 2)$ distante da extremidade anterior. Esôfago medindo 894,34 $(\mathrm{n}=2)$ de comprimento, 129,08 e $147,52(138,3 \pm 13,03 ; 2)$ de largura, correspondendo a $7,87 \%$ do CTC. Espículos desiguais, o maior com 433,34 $(\mathrm{n}=2)$ e o menor com 387,24 e 414,9 (401,7 $\pm 19,55$; 2). Gubernáculo com 75,52 e $80,24(77,88 \pm 3,33$; 2) de comprimento, 16,52 e $20,16(18,34 \pm 2,57 ; 2)$ de largura. Ventosa circular, pré-cloacal, medindo 64,54 e $73,76(69,15 \pm 6,51 ; 2)$ de diâmetro, 424,12 e $433,34(428,73 \pm 6,51 ; 2)$ distante da extremidade posterior. Papilas caudais distribuídas em 21 pares, da região da ventosa até extremidade da região posterior, sendo sete pares pré-cloacais e 14 pares pós-cloacais. 
FIGURAS 2-5: Fotomicrografias de Paraspidodera uncinata (Rudolphi, 1819) Travassos, 1914 coletadas de Cavia aperea Erxleben, 1777 e Cavia magna Ximenez, 1980.

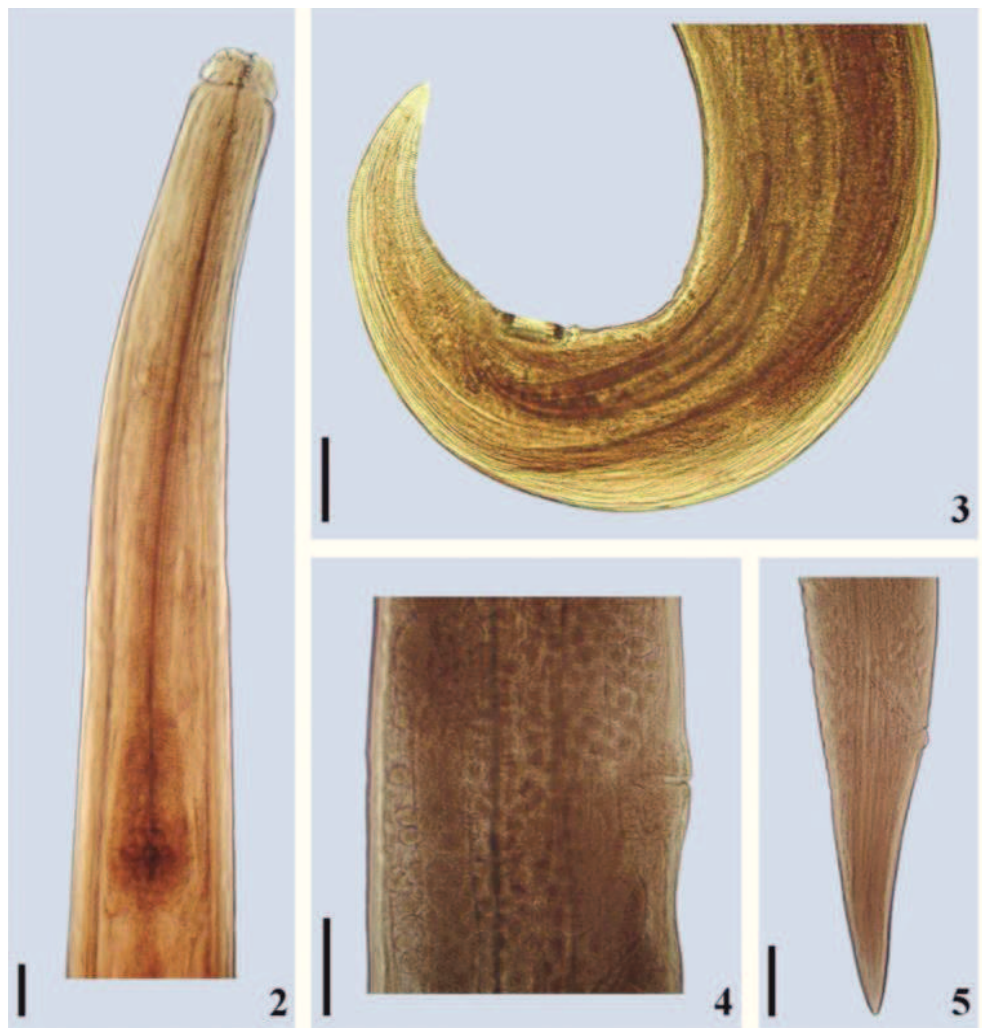

(2) Região anterior do macho. Barra $=150 \mu \mathrm{m}$. (3) Região posterior do macho. Barra $=100 \mu \mathrm{m}$. (4) Região vulvar da fêmea. Barra $=100$ $\mu \mathrm{m}$. (5) Região posterior da fêmea. Barra $=150 \mu \mathrm{m}$.

Cloaca situada a 295,04 e $322,7(308,87 \pm 19,55 ; 2)$ da extremidade posterior.

Fêmeas $(\mathrm{n}=6)$. Corpo com 12,42-15,50 mm $(14,13$ $\pm 1,03 ; 6)$ de comprimento, 285,82-341,14 (318,09 \pm $19,11 ; 6)$ de largura. Lábios medindo 101,42-119,86 $(107,56 \pm 7,52 ; 6)$ de comprimento, 64,54-73,76 (66,07 $\pm 3,76 ; 6)$ de largura. Esôfago medindo 931-1051 $(986,25 \pm 55,98 ; 4)$ de comprimento, 92,2-165 (128,55 $\pm 30,71 ; 4)$ de largura, correspondendo a $6,93 \%$ do CTC. Poro excretor a $606,52-698,56(652,54 \pm 65,08 ; 2) d a$ extremidade anterior. Vulva 626,96-682,28 (654,62 \pm $39,11 ; 2)$ distante da região anterior. Ovos com 55,32$64,54(56,85 \pm 3,76 ; 6)$ de comprimento, 36,88-46,1 $(41,49 \pm 5,05 ; 6)$ de largura. Ânus situado a 553,2-663,84 $(614,45 \pm 45,98 ; 6)$ da extremidade posterior.

\section{Resenha taxonômica}

Sinônimos: Ascaris uncinata Rudolphi, 1819; Heterakis uncinata Schneider, 1866; Subulura uncinata
Hall, 1916; Paraspidodera americana Khalil \& Vogelsang, 1931; Paraspidodera uruguaya Khalil \& Vogelsang, 1931.

Hospedeiros: Cavia aperea e Cavia magna - novo registro de hospedeiro.

Espécimes depositados: MCNU 1319 (C. aperea) e MCNU 24523 (C. magna).

Localidades: Pelotas, Bagé (BR 116) e Maratá ( $C$. aperea); Ilha dos Marinheiros, Rio Grande (C. magna), RS, Brasil.

Locais das infecções: Intestino grosso (C. aperea); intestino delgado e grosso (C. magna).

Prevalências: 14\% (C. aperea) e 60\% (C. magna).

Intensidade Média das Infecções: 6 (C. aperea) e 3,6 helmintos/hospedeiro (C. magna).

Abundância Média das Infecções: 0,85 (C. aperea) e 2,2 helmintos/hospedeiro (C. magna). 
Amplitude da intensidade de infecção: 1-6 (C. aperea) e 1-9 (C. magna).

Espécimes representativos de helmintos depositados: CHMU 70-1-1 (macho); CHMU 138-1-1 (fêmea).

\section{Discussão}

A distinção entre as espécies de Paraspidodera e Aspidodera Railliet \& Henry, 1912 é baseada nos cordões na extremidade cefálica e lobos laterais dos lábios (TRAVASSOS, 1914; CHABAUD, 2009). A ausência de cordões e lobos laterais dos lábios simples permitiram a identificação dos espécimes como $P$. uncinata.

Os espécimes examinados por Pereira e Vaz (1933), Sutton (1976) e Rossin et al. (2004) são maiores em relação aos espécimes examinados no presente estudo. A medida da distância da vulva em relação à região anterior $(654,62)$ de $P$. uncinata foi menor do que registrado por Pinto et al. (2002) (734,2). Entretanto, os espécimes examinados no presente estudo são menores $(12,42-15,5$ $\mathrm{mm}$ ) em relação aos espécimes medidos (15-20 mm) por Pinto et al. (2002). Nos espécimes analisados, não foi possível visualizar o anel nervoso.

A medida da distância do ânus em relação à extremidade posterior registrada por Travassos (1914) $(1,198 \mathrm{~mm})$ é superior ao encontrado no presente estudo $(614,45)$ e nos demais trabalhos. Esta diferença possivelmente está relacionada a um erro de medida, pois o trabalho de Travassos (1914) não possui representação gráfica da fêmea para comparação, além disso, nos diferentes estudos os comprimentos das fêmeas e os comprimentos das caudas são semelhantes como o registrado no presente estudo.

Em relação às prevalências de $P$. uncinata, existem diferenças registradas na literatura, considerando o hospedeiro e tamanho amostral: Dittmar (2002) em $C$. aperea $(\mathrm{n}=143)$ encontrou uma prevalência de $37 \%$; Almeida (2004) em K. rupestris $(\mathrm{n}=16)$ registrou prevalência de 31\%; Krüger (2006) registrou em $C$. aperea $(\mathrm{n}=33)$ prevalência de $76 \%$ (nos cecos) e $48 \%$ (no intestino grosso); Rossin et al. (2004) registrou em
Ctenomys talarum Thomas, $1898(\mathrm{n}=81)$ prevalência de $93,8 \%$. No presente estudo foram observadas prevalências de $60 \%(C$. magna; $\mathrm{n}=5)$ e $14 \%(C$. aperea $; \mathrm{n}=7)$. Estas diferenças encontradas podem estar relacionadas ao número de hospedeiros examinados.

Em relação às diferenças entre as prevalências das espécies examinadas neste estudo, parecem estar relacionadas aos hábitos de forrageio. A prevalência encontrada para C. magna foi superior e reflete a ingestão de ovos do parasito (ANDERSON, 2000) em vegetais nos ambientes naturais, contrastando com C. aperea, cujos hábitos são descritos na literatura (SILVA et al., 2013) como uma espécie que se alimenta em beiras de rodovias, portanto ingerindo mais grãos (liberados dos carregamentos de caminhões) além de ocupar um ambiente antropizado. $\mathrm{O}$ encontro de $P$. uncinata nas espécies de preás (C. porcellus, C. aperea e C. magna) permite inferir que esses roedores apresentam o modo de forrageio semelhante, entretanto, apresentam diferenças na quantidade de vegetais com ovos ingeridos nas diferentes regiões do RS. Até o presente momento, não existe nenhuma espécie de parasito registrada para C. magna, portanto este estudo contribui com o conhecimento da biodiversidade parasitária de preás e registra pela primeira vez $P$. uncinata em $C$. magna no Rio Grande do Sul, Brasil.

\section{Agradecimentos}

Ao Laboratório de Sistemática e Evolução de Mamíferos, MCNU, pela doação dos hospedeiros. Ao Professor Dr. Stephen F. Ferrari, UFS, pela revisão do Abstract.

\section{Referências}

ALMEIDA, K. S. Helmintos parasitos de mocós (Kerodon rupestris Wied, 1820), de vida livre e de cativeiro, criados no semi-árido nordestino. 2004. 41 f. Dissertação (Mestrado em Medicina Veterinária) - Universidade Estadual Paulista, São Paulo. 2004.

AMATO, J. F. R.; AMATO, S. B. Técnicas gerais para coleta e preparação de helmintos endoparasitos de aves. In: VON MATTER, S.; STRAUBE, F. C.; ACCORDI, I. A.; PIACENTINI, V. Q.; CÂNDIDO-JR, J. F. (Org.). Ornitologia e conservação: ciência aplicada, técnicas de pesquisa e levantamento. Rio de Janeiro: Technical Books, 2010. p. 369-393. 
ANDERSON, R. C. Nematode parasites of vertebrates: their development and transmission. 2. ed. Wallingford: CABI Publishing, 2000. $650 \mathrm{p}$.

BUSH, A. O.; LAFFERTY K. D.; LOTZ, J. M.; SHOSTAK, A. W. Parasitology meets ecology on its own terms: Margolis et al. revisited. Journal of Parasitology, Lawrence, v. 83, n. 4, p. 575583, 1997.

CHABAUD, A. G. Ascaridida: Cosmocercoidea, Seuratoidea and Heterakoidea. In: ANDERSON, R. C.; CHABAUD, A. G.; WILLMOTT, S. (Ed.). Keys to the Nematode parasites of vertebrates. Wallingford: CABI, 2009. p. 248-308.

CHEREM, J. J.; FERIGOLO, J. Descrição do sincrânio de Cavia aperea (Rodentia, Caviidae) e comparação com as demais espécies do gênero no Brasil. Papéis Avulsos de Zoologia, São Paulo, v. 52, n. 3, p. 21-50, 2012.

DITTMAR, K. Arthropod and helminth parasites of the wild guinea pig, Cavia aperea, from the Andes and the cordillera in Peru, South America. Journal of Parasitology, Lawrence, v. 88, n. 2, p. 409411, 2002.

FERNANDES, F. A.; QUINTELA, F. M.; FERNÁNDEZ, G. P.; GONÇALVES, G. L. Os roedores. In: GONÇALVES, G. L.; QUINTELA, F. M.; FREITAS, T. R. O. (Org.). Mamíferos do Rio Grande do Sul. Porto Alegre: Pacartes, 2014. p. 55-87.

HUMASON, G. L. Animal tissue techniques. 4 ed. São Francisco: W. H. Freeman and Company, 1979. 661 p.

IBÁÑEZ, N. H. Helminthologic fauna of Peru. Paraspidodera uncinata (Rudolphi, 1819), intestinal parasite of guinea pigs (Nematoda, Subuluroidea). Boletín Chileno di Parasitología, Santiago do Chile, v. 22, n. 1, p. 15-20, 1967.

KAWAZOE, U.; CORDEIRO, N. S.; ARTIGAS, P. T. Taxorchis caviae sp. n. (Trematoda, Paramphistomidae), parasito intestinal de Cavia aperea aperea Erxleben, 1777 (Rodentia, Caviidae). Memórias do Instituto Oswaldo Cruz, Rio de Janeiro, v. 76, n. 1, p. 1-13, 1981.

KHALIL, M.; VOGELSANG, E. G. Paraspidodera americana n. sp. parasitic in a South American rodent. Zeitschrift für Parasitenkunde, Berlin, v. 3, n. 2, p. 142-144, 1931a.

KHALIL, M.; VOGELSANG, E. G. On a new species of Paraspidodera, P. uruguaya sp. n. Zeitschrift für Parasitenkunde, Berlin, v. 3, n. 2, p. 145-147, 1931 b.

KRÜGER, C. P. Artrópodes e helmintos parasitos de Cavia aperea Exerleben, 1777 (Rodentia: Caviidae) no sul do Brasil. 2006. 68 f. Dissertação (Mestrado em Parasitologia) - Universidade Federal de Pelotas, Pelotas. 2006.

LENT, H.; FREITAS, J. F. T. Uma coleção de nematódeos, parasitos de vertebrados, do Museu de Historia Natural de Montevideo. Memórias do Instituto Oswaldo Cruz, Rio de Janeiro, v. 46, n. 1, p. 1-71, 1948.

MMA - MINISTÉRIO DO MEIO AMBIENTE. Instrução Normativa $\mathrm{n}^{\mathrm{o}} 154$ de 01 de março de 2007. Diário Oficial da União, Brasília, n. 42, p. 57-59, 2007.

OLIVEIRA, J. A.; BONVICINO, C. R. Ordem Rodentia. In: REIS, N. R.; PERACCHI, A. L.; PEDRO, W. A.; LIMA, I. P. (Ed.). Mamíferos do Brasil. 2. ed. Londrina: Nélio R. dos Reis, 2011. p. 358-414.

PARONA, C. Di alcuni elminti del Museo Nacional di Buenos Aires. Comunicaciones del Museo Nacional de Buenos Aires, Buenos Aires, v. 1, p. 190-197, 1900.
PEREIRA, C.; VAZ, Z. Nota sobre a presença de Paraspidodera uncinata, em cobayas de São Paulo. Revista de Biologia e Higiene, São Paulo, v. 2, p. 52-55, 1933.

PINTO, R. M.; GOMES, D. C.; MUNIZ-PEREIRA, L; NORONHA, D. Helminths of the guinea pig, Cavia porcellus (Linnaeus), in Brazil. Revista Brasileira de Zoologia, Curitiba, v. 19, supl. 1, p. 261-269, 2002.

PINTO, R. M.; KOHN, A.; FERNANDES, B. M. M.; MELLO, D. A. Nematodes of rodents in Brazil, with description of Aspidodera vicentei n. sp. Systematic Parasitology, Dordrecht, v. 4, p. 263267, 1982.

REGO, A. A. Revisão do gênero Monoecocestus Beddard, 1914 (Cestoda, Anoplocephalidae). Memórias do Instituto Oswaldo Cruz, Rio de Janeiro, v. 59, n. 3, p. 325-354, 1961.

REY, L. Non-human vertebrate hosts of Schistosoma mansoni and schistosomiasis transmission in Brazil. Research and Reviews in Parasitology, Barcelona, v. 53, n. 1-2, p. 13-25, 1993.

RIBEIRO, B. P.; MÜLLER, G.; COSTA, P. R. P.; WIEGAND, M. M. Paraspidodera uncinata (Rud.,1819) Travassos, 1914 em Cavia porcellus no município do Capão do Leão, Rio Grande do Sul, Brasil. Arquivo Brasileiro de Medicina Veterinária e Zootecnia, Belo Horizonte, v. 37, n. 4, p. 377-379, 1985.

ROBLES, M. R.; GALLIARI, C.; NAVONE, G. T. New records of nematode parasites from Euryzygomatomys spinosus (Rodentia, Echimyidae) in Misiones province, Argentina. Mastozoología Neotropical, Mendoza, v. 19, n. 2, p. 163-178, 2012.

ROSSIN, M. A.; TIMI, J. T.; MALIZIA, A. I. Redescription and new host record of Parapidodera uncinata (Rudolphi, 1819) (Nematoda, Aspidoderidae) from the South American subterranean rodent Ctenomys talarum (Rodentia, Octodontidae). Acta Parasitologica, Warsaw, v. 49, n. 4, p. 325-333, 2004.

SILVA, D. E.; CORRÊA, L. L. C.; OLIVEIRA, S. V.; CAPPELLARI, L. H. Monitoramento de vertebrados atropelados em dois trechos de rodovias na região central do Rio Grande do Sul-Brasil. Revista de Ciências Ambientais, Canoas, v. 7, n. 1, p. 27-36, 2013.

SIMÕES, R.; GENTILE, R.; RADEMAKER, V.; D'ANDREA, P.; HERRERA, H.; FREITAS, T.; LANFREDI, R.; MALDONADO JR., A. Variation in the helminth community structure of Thrichomys pachyurus (Rodentia: Echimyidae) in two sub-regions of the Brazilian Pantanal: the effects of land use and seasonality. Journal of Helminthology, London, v. 84, n. 3, p. 266-275, 2010.

SUTTON, C. A. Contribución al conocimiento de la fauna parasitaria Argentina III. Endoparásitos de Cavia aperea pamparum Thomas. Neotropica, La Plata, v. 22, p. 33-40, 1976.

TRAVASSOS, L. Contribuição para o conhecimento da fauna helmintolojica brazileira. Novo genero da familia Heterakidae (Railliet \& Henry). Memórias do Instituto Oswaldo Cruz, Rio de Janeiro, v. 6, n. 2, p. 137-142, 1914.

VICENTE, J. J.; GOMES, D. C.; ARAUJO FILHO, N. A. Alguns helmintos de marsupiais e roedores da Ilha Grande, estado do Rio de Janeiro. Atas da Sociedade de Biologia do Rio de Janeiro, Rio de Janeiro, v. 23, p. 3-4, 1982.

VICENTE, J. J.; RODRIGUES, H. O.; GOMES, D. C.; PINTO, R. M. Nematóides do Brasil. Parte V: Nematóides de mamíferos. Revista Brasileira de Zoologia, Curitiba, v. 14, supl. 1, p. 1-452, 1997. 